\title{
Analysis of Irreversible Processes across Narrow Junctions
}

\author{
J.M. HONIG* AND R. HOEHN \\ Department of Chemistry, Purdue University, West Lafayette, Indiana, 47907, USA
}

(Received November 17, 2010)

\begin{abstract}
The rectification of the second law of thermodynamics is used to directly relate irreversible heat and work transfers to reversible processes. This permits the construction of thermodynamic functions of state that include entropy contributions due to irreversible processes. A general expression is set up to determine the entropy changes in terms of experimentally accessible parameters when a system is interacting with its surroundings via quasistatic irreversible operations. The procedure is used to determine the entropy changes across a narrow junction in terms of pressure and temperature differences between the system and its surroundings, including cyclic processes.
\end{abstract}

PACS: 05.70.--a, 05.70.Ln, 44.10.+i

\section{Introduction}

This article deals with the thermodynamic analysis of irreversible processes across a narrow interface which separates a system from its surroundings (reservoir), the combination forming an isolated unit. We first develop the required fundamental relations that are scattered in the literature and then show how they are applied in specific situations.

At the outset we clarify what is meant by intensive variables that generate irreversible changes. Reference is made to Fig. 1 which illustrates the temperature profile for the compound system under conditions described below. Similar considerations apply to all other intensive variables of interest. The reservoir is assumed to be so large that under all operating conditions its temperature, $T_{0}$, remains constant and uniform over almost its entire extension. The temperature then changes across the small interfacial region, which is a poor thermal conductor, to its value $T$ of the system. In conformity with standard operating procedures we assume all processes in the reservoir to proceed reversibly. All irreversible changes within the system are assumed to proceed sufficiently slowly that its temperature $T$ is uniformly variable over almost the entire extension of the system. Such operations will be termed quasistatic irreversible processes (QSIPs). The system and surroundings are separated by a narrow interface over which the temperature changes from $T_{0}$ to $T$. There is no restriction on the difference between $T$ and $T_{0}$.

The present approach, dealing with entropy changes in irreversible processes, is an extension of earlier work [1-7], and is complementary to the standard theory of ir-

* corresponding author; e-mail: jmh@purdue.edu

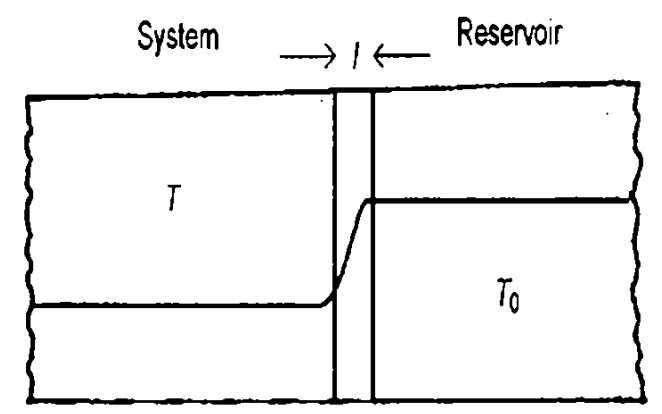

Fig. 1. Sketch of temperature profile for the combined system and reservoir at different temperatures $T$ and $T_{0}$. The temperature in each phase remains essentially constant over almost the entire region; the gradient in temperature develops over only a small region $l$ at the interface.

reversible thermodynamics (e.g., [8-10]) that emphasizes the relation between fluxes and forces.

\section{Fundamentals}

We first provide a fundamental analysis of irreversible phenomena that is used below. Consider an infinitesimal step in a process that is carried out first reversibly $(a)$ and then irreversibly $(b)$. For the compound unit the entropy change in the two cases is given by

$$
\begin{aligned}
& \text { and } S+\mathrm{d}_{a} S_{0}=0 \\
& \text { d } S+\mathrm{d}_{b} S_{0}>0,
\end{aligned}
$$

where the zero subscript refers to the surroundings, and where the entropy change in the system is identical in the two cases, since $S$ is a function of state of the system. The use of inequalities is awkward; hence, it is 
apposite to introduce an entropy deficit function $\mathrm{d} \theta>0$ that converts Eq. (2) to an equality

$$
\mathrm{d} S+\mathrm{d}_{b} S_{0}-\mathrm{d} \theta=0 .
$$

Let us note that $\mathrm{d} \theta$ is the sum of the entropy changes in the system plus surroundings kept under different conditions; it represents the total entropy change of the universe resulting from the execution of the irreversible process.

We next set $\mathrm{d} S=\mathrm{d}_{\mathrm{r}} Q / T$, where $\mathrm{d}_{\mathrm{r}} Q$ is the element of heat reversibly absorbed (or released) by the system at temperature $T$. Since all processes in the reservoir take place reversibly we designate the response of the reservoir as $\mathrm{d}_{b} S_{0}=\mathrm{d} Q_{0} / T_{0}=-\mathrm{d}_{i} Q / T_{0}$. The second equality is obtained because of the isolation of the compound system; heat gained (lost) by the system is lost (gained) by the reservoir.

\section{Heat transfer}

When $\mathrm{d} S$ and $\mathrm{d}_{b} S_{0}$ in Eq. (3) are replaced as described above one may relate the heat transferred irreversibly to the heat transferred reversibly as follows:

$$
\mathrm{d}_{i} Q=\left(T_{0} / T\right) \mathrm{d}_{\mathrm{r}} Q-T_{0} \mathrm{~d} \theta<\left(T_{0} / T\right) \mathrm{d}_{\mathrm{r}} Q .
$$

This relation was previously derived in Ref. [11]. The inequality may be tightened by noting that it must hold for any value $T<T_{0}$ when heat is absorbed by the system and for any $T>T_{0}$ when heat is released; in either case the limiting temperature is $T=T_{0}$. One thus arrives at the string of inequalities

$$
\mathrm{d}_{i} Q<\mathrm{d}_{\mathrm{r}} Q<\left(T_{0} / T\right) \mathrm{d}_{\mathrm{r}} Q,
$$

which either becomes more positive or less negative from left to right. The result $\mathrm{d}_{i} Q<\mathrm{d}_{\mathrm{r}} Q$ is simply a reformulation of the famous Clausius inequality.

It is expedient to rewrite Eq. (4) in the form

$$
\mathrm{d}_{i} Q=\mathrm{d}_{\mathrm{r}} Q+\left(T_{0} / T-1\right) \mathrm{d}_{\mathrm{r}} Q-T_{0} \mathrm{~d} \theta .
$$

Because of the Clausius inequality the last two terms in (6) must be negative. This sets up a lower bound on $\mathrm{d} \theta$ :

$$
\text { d } \theta>\left[\left(T_{0}-T\right) / T_{0} T\right] \mathrm{d}_{\mathrm{r}} Q=\left[\left(T_{0}-T\right) / T_{0}\right] \mathrm{d} S>0,(7)
$$

which is positive because the quantity in square brackets has the same sign as $\mathrm{d} S$ or $\mathrm{d}_{\mathrm{r}} Q$, all being positive or negative according as heat is incrementally introduced into or withdrawn from the system. The quantities on the right in principle are experimentally accessible.

\section{Work performance}

Information on work performance is accessed through the first law of thermodynamics in the form

$$
\mathrm{d} E=\mathrm{d}_{\mathrm{r}} Q+\mathrm{d}_{\mathrm{r}} W=\mathrm{d}_{i} Q+\mathrm{d}_{i} W,
$$

where $E$ is the energy of the system and $W$ indicates the work performance. On substituting from (6) one obtains a relation between work performed reversibly and irreversibly as

$$
\begin{gathered}
\mathrm{d}_{i} W=\mathrm{d}_{\mathrm{r}} W-\left(T_{0} / T-1\right) \mathrm{d}_{\mathrm{r}} Q+T_{0} \mathrm{~d} \theta \\
=\mathrm{d}_{\mathrm{r}} W-\left(T_{0}-T\right) \mathrm{d} S+T_{0} \mathrm{~d} \theta
\end{gathered}
$$

where the last two terms are opposite in sign to those in Eq. (6). It then follows that $\mathrm{d}_{i} W>\mathrm{d}_{\mathrm{r}} W$, known as the Gouy-Stodola theorem.

Equation (9) may be rewritten in the form

$$
\mathrm{d} \theta=\left(\mathrm{d}_{i} W-\mathrm{d}_{\mathrm{r}} W\right) / T_{0}+\left(1-T / T_{0}\right) \mathrm{d} S .
$$

This provides one avenue for determining the incremental contribution of an irreversible process to the entropy of the universe. The quantities on the right are accessible by measurement or calculation. The special cases $\mathrm{d} \theta=$ $\left(1-T / T_{0}\right) \mathrm{d} S$ as well as $\mathrm{d} \theta=\left(\mathrm{d}_{i} W-\mathrm{d}_{\mathrm{r}} W\right) / T_{0}$ were derived by independent methods in Refs. [12] and [13], respectively.

Here we concentrate on an alternative formulation that provides similar information in terms of the physical properties of the system and its surroundings, as we now show.

\section{Functions of state}

The energy differential for the surroundings takes the customary form

$$
\mathrm{d} E_{0}=T_{0} \mathrm{~d} S_{0}-P_{0} \mathrm{~d} V_{0}+\Sigma_{i} \mu_{0 i} \mathrm{~d} n_{0 i},
$$

where $P_{0}$ is the prevailing pressure, $V_{0}$ - the volume, $\mu_{0 i}$ - the chemical potential of species $i$, and $n_{0 i}$ - the mole number of species i, all referred to the surroundings, functioning reversibly. We now set $\mathrm{d} E=-\mathrm{d} E_{0}$ and $\mathrm{d} n_{0 i}=-\mathrm{d} n_{i}$, where the nonsubscripted quantities refer to the system. We further assume that any volume change of the system is precisely compensated for by the volume change in the surroundings, so that $\mathrm{d} V_{0}=-\mathrm{d} V$. Lastly, we introduce Eq. (3) by setting $\mathrm{d} S_{0} \equiv \mathrm{d}_{b} S_{0}=-\mathrm{d} S+\mathrm{d} \theta$. This leads to the fundamental expression for the energy of the system in the form

$$
\mathrm{d} E=T_{0} \mathrm{~d} S-P_{0} \mathrm{~d} V+\Sigma_{i} \mu_{0 i} \mathrm{~d} n_{i}-T_{0} \mathrm{~d} \theta,
$$

thereby generalizing the conventional relation for the energy differential, so that it may be applied to QSIP processes. This relation was established earlier by Kestin [12], using a different technique. One should note that even for irreversible processes of the system all intensive variables refer to the properties of the surroundings.

For future use it is expedient to rewrite Eq. (12) as

$$
\begin{aligned}
& \mathrm{d} E=\left(T_{0}-T\right) \mathrm{d} S-\left(P_{0}-P\right) \mathrm{d} V+\Sigma_{i}\left(\mu_{0 i}-\mu_{i}\right) \mathrm{d} n_{i} \\
& \quad+T \mathrm{~d} S-P \mathrm{~d} V+\Sigma_{i} \mu_{i} \mathrm{~d} n_{i}-T_{0} \mathrm{~d} \theta,
\end{aligned}
$$

from which we subtract the same energy differential, $\mathrm{d} E$, for the same step executed reversibly,

$$
\mathrm{d} E=T \mathrm{~d} S-P \mathrm{~d} V+\Sigma_{i} \mu_{i} \mathrm{~d} n_{i} .
$$

This permits us to solve for 


$$
\begin{aligned}
& \mathrm{d} \theta=\left(1 / T_{0}\right)\left[\left(T_{0}-T\right) \mathrm{d} S-\left(P_{0}-P\right) \mathrm{d} V\right. \\
& \left.\quad+\Sigma_{i}\left(\mu_{0 i}-\mu_{i}\right) \mathrm{d} n_{i}\right] .
\end{aligned}
$$

We have thereby expressed the contribution of the infinitesimal step to the entropy generated in executing a QSI process. Equation (15) is consistent with Eq. (3).

For further progress it is expedient to introduce $T$, $P$, and $n_{i}$ as control variables, thereby eliminating the entropy and volume differentials. This is accomplished by specifying $S=S\left(T, P, n_{i}\right)$ and $V=V\left(T, P, n_{i}\right)$ and then taking differentials

$$
\begin{aligned}
\mathrm{d} S & =\frac{\partial S}{\partial T} \mathrm{~d} T+\frac{\partial S}{\partial P} \mathrm{~d} P+\sum_{i} \frac{\partial S}{\partial n_{i}} \mathrm{~d} n_{i} \\
& =\frac{C_{P, n}}{T} \mathrm{~d} T-\frac{\partial V}{\partial T} \mathrm{~d} P+\sum_{i} \bar{S}_{i} \mathrm{~d} n_{i},
\end{aligned}
$$

where we have inserted the heat capacity $C_{\mathrm{P}, \mathrm{n}}$ at constant pressure and composition, the relevant Maxwell relation, and the partial molal entropy, $\bar{S}_{i}$. We obtain similarly

$$
\mathrm{d} V=\frac{\partial V}{\partial T} \mathrm{~d} T+\frac{\partial V}{\partial P} \mathrm{~d} P+\sum_{i} \bar{V}_{i} \mathrm{~d} n_{i} .
$$

When these relations are substituted in (15) we generate the fundamental working equation

$$
\begin{aligned}
\mathrm{d} \theta & =\left[\left(1-\frac{T}{T_{0}}\right) \frac{C_{P}}{T}-\frac{1}{T_{0}}\left(P_{0}-P\right) \frac{\partial V}{\partial T}\right] \mathrm{d} T \\
- & {\left[\left(1-\frac{T}{T_{0}}\right) \frac{\partial V}{\partial T}+\frac{1}{T_{0}}\left(P_{0}-P\right) \frac{\partial V}{\partial P}\right] \mathrm{d} P } \\
& +\sum_{i}\left[\left(1-\frac{T}{T_{0}}\right) \bar{S}_{i}-\frac{1}{T_{0}}\left(P_{0}-P\right) \bar{V}_{i}\right. \\
& \left.+\frac{1}{T_{0}}\left(\mu_{0 i}-\mu_{i}\right)\right] \mathrm{d} n_{i} .
\end{aligned}
$$

\section{Special cases}

In the above we have specified the incremental contribution to the entropy associated with irreversible quasistatic processes taking place across the interface, in terms of temperature, pressure, and composition of the system as the proper control variables. To obtain the total contribution we must now specify how each of the nonsubscripted variables changes with time $t$ in the overall process, and then integrate the rather formidable expression. For illustrative purposes we therefore introduce a number of reasonable simplifications and special cases that show how to implement the calculation.

We assume that the reservoir is of such huge size that all its variables do not change appreciably during the interaction with the system; then the subscripted quantities are constant. We next specialize to the case of a one-component system at constant composition, which eliminates the third line of Eq. (18). We further divide the calculations into two groups: (a) Systems consisting of an ideal gas, for which $V=n R T / P$ and $C_{P}=5 n R / 2$, where $n$ is the number of moles of material in the system and $R$ is the gas constant. (b) Condensed phases, where we simulate the modest changes of $V$ with $T$ and $P$ by replacing $\partial V / \partial T$ with $\alpha n \tilde{V}_{0}$, and $\partial V / \partial P$, with $-\beta n \tilde{V}_{0}$. Here $\alpha$ is the isobaric coefficient of thermal expansion, $\beta$ is the isothermal coefficient of compression, and $\tilde{V}_{0}$ is a suitably averaged molar volume over the temperature and pressure range of interest. All three of these quantities are assumed to be constant. Lastly, to be definite, we set $T \leq T_{0}$ and $P \leq P_{0}$, so that, as the interaction is turned on, the temperature and pressure of the system rises. The volume of the system adjusts in conformity with the equation of state of the material.

(a) For ideal gases the calculation may be subdivided into two categories: integrals that are independent of path, and line integrals that involve more than one variable. In the former case the time dependence is irrelevant. On substituting in (18) for $C_{P}$, determining the volume derivatives, and integrating the differentials that involve only one independent variable ( $T$ or $P$ ) between limits $\mathrm{i}$ and $\mathrm{f}$ we obtain

$$
\theta_{a \mathrm{i}}=\frac{5}{2} n R \ln \frac{T_{\mathrm{f}}}{T_{\mathrm{i}}}-\frac{3}{2} n R \ln \frac{1}{T_{0}}\left(T_{\mathrm{f}}-T_{\mathrm{i}}\right)-n R \ln \frac{P_{\mathrm{f}}}{P_{\mathrm{i}}} .
$$

Here the first and last terms are those encountered for the reversible alteration of temperature and pressure of an ideal gas.

To handle the remainder it is necessary to introduce time as a parameter in the interval $0 \leq t \leq \tau$ and to deal with the line integrals in the form

$$
\begin{aligned}
\theta_{a d} & \equiv \theta_{T}+\theta_{P}=-\frac{P_{0}}{T_{0}} n R \int_{0}^{\tau} \frac{1}{P(t)} \frac{\mathrm{d} T}{\mathrm{~d} t} \mathrm{~d} t \\
+ & \frac{P_{0}}{T_{0}} n R \int_{0}^{\tau} \frac{T(t)}{P^{2}(t)} \frac{\mathrm{d} P}{\mathrm{~d} t} \mathrm{~d} t .
\end{aligned}
$$

We now take up two special examples:

Case 1. We set $P=P_{\mathrm{i}} \mathrm{e}^{k_{P} t}, T=T_{\mathrm{i}} \mathrm{e}^{k_{T} t}, 0 \leq t \leq \tau$, where $k_{P}, k_{T}$ are time constants, so chosen that at time $\tau$ the system has reached the final values $T_{\mathrm{f}}$ and $P_{\mathrm{f}}$. On inserting these functions into (20) the integrations are straightforward, though lengthy. We obtain

$$
\begin{aligned}
\theta_{T} & =-\frac{P_{0}}{T_{0}} \frac{T_{\mathrm{i}}}{P_{\mathrm{i}}} n R \frac{1}{1-k_{P} / k_{T}}\left(\frac{T_{\mathrm{f}} / T_{\mathrm{i}}}{P_{\mathrm{f}} / P_{\mathrm{i}}}-1\right), \\
\theta_{P} & =\frac{P_{0}}{T_{0}} \frac{T_{\mathrm{i}}}{P_{\mathrm{i}}} n R \frac{1}{k_{T} / k_{P}-1}\left(\frac{T_{\mathrm{f}} / T_{\mathrm{i}}}{P_{\mathrm{f}} / P_{\mathrm{i}}}-1\right), \\
\frac{k_{P}}{k_{T}} & =\frac{\ln \left(P_{\mathrm{f}} / P_{\mathrm{i}}\right)}{\ln \left(T_{\mathrm{f}} / T_{\mathrm{i}}\right)},
\end{aligned}
$$

which are combined to yield

$$
\theta=n R \frac{P_{0} T_{\mathrm{i}}}{P_{\mathrm{i}} T_{0}}\left(1-\frac{P_{\mathrm{i}} T_{\mathrm{f}}}{P_{\mathrm{f}} T_{\mathrm{i}}}\right)=n R \frac{P_{0}}{T_{0}}\left(\frac{T_{\mathrm{i}}}{P_{\mathrm{i}}}-\frac{T_{\mathrm{f}}}{P_{\mathrm{f}}}\right),
$$

to which we adjoin Eq. (19). According to Eq. (3), the 
resulting expression specifies the entropy increase in the universe arising from the irreversible QSIP interactions between the ideal gas in the system and in the reservoir. Let us note that in addition to the properties of the reservoir only initial and final values of the temperature and pressure of the system appear in the above results.

Case 2. We next take up the particular time dependence $P=P_{\mathrm{i}} \mathrm{e}^{k_{P} t}, T=T_{\mathrm{i}}\left(1+k_{T} t\right), 0 \leq t \leq \tau$. Obviously, there is no change in handling the one-variable integrals. Proceeding in the same manner with the path-dependent integrals we obtain the expressions

$$
\begin{aligned}
& \theta_{T}=n R \frac{P_{0} T_{\mathrm{i}}}{P_{\mathrm{i}} T_{0}} \frac{k_{T}}{k_{P}}\left(\frac{P_{\mathrm{i}}}{P_{\mathrm{f}}}-1\right), \\
& \theta_{P}=n R \frac{P_{0} T_{\mathrm{i}}}{P_{\mathrm{i}} T_{0}}\left[\left(1-\frac{P_{\mathrm{i}}}{P_{\mathrm{f}}}\right)+\frac{k_{T}}{k_{P}}\left(1-\frac{P_{\mathrm{i}}}{P_{\mathrm{f}}}+\frac{P_{\mathrm{i}}}{P_{\mathrm{f}}} \ln \frac{P_{\mathrm{i}}}{P_{\mathrm{f}}}\right)\right], \\
& \frac{k_{T}}{k_{P}}=\frac{T_{\mathrm{f}} / T_{\mathrm{i}}-1}{\ln \left(P_{\mathrm{f}} / P_{\mathrm{i}}\right)},
\end{aligned}
$$

which clearly differ from Eq. (21). Nevertheless, on summing these expressions one again recovers Eq. (22) identically. This demonstrates that while the contributions to $\theta_{P}$ and to $\theta_{T}$ differ for the two cases their sum is independent of the choice for the time variation of $T$ and of $P$. This fact may be rationalized by noting that in the QSIP approximation there is no reference to the path that leads from the initial to the final state. This holds true as long as relaxation effects are ignored. The reader may also verify our finding that a choice in which both $P$ and $T$ vary linearly with time produces yet a different set of $\theta_{P}$ and $\theta_{T}$ integrals that again sum to Eq. (22). Thus, Eqs. (19) and (22) are the final results of interest for the ideal gas.

(b) For the condensed phase we replace the partial derivatives of $V$ in Eq. (18), using the equation of state for condensed phases in the form

$$
\begin{aligned}
V & =V_{0}+\int(\partial V / \partial T) \mathrm{d} T+\int(\partial V / \partial P) \mathrm{d} P \\
& =V_{0}(1+\alpha T-\beta P),
\end{aligned}
$$

where we later retain the correction terms only to first order in $\alpha$ and $\beta$. We then utilize the caloric equation of state for the enthalpy $H$ of the condensed phase in the form

$$
(\partial H / \partial T)=V-T(\partial V / \partial T)=V_{0}(1-\beta P),
$$

which yields the integrated form as

$$
H=H_{0}+V_{0} P-(1 / 2) \beta V_{0} P^{2} .
$$

Here $H_{0}=3 n R T$ is the arbitrary function of integration for the condensed phase. Accordingly, the heat capacity to first order of smallness is given by $C_{P}=3 n R$.

We now return to the first line of Eq. (18) and first single out those differentials that involve only one independent variable. On performing that integration we obtain

$$
\begin{aligned}
\theta_{b \mathrm{i}} & =n \tilde{C}_{P} \ln \frac{T_{\mathrm{f}}}{T_{\mathrm{i}}}-n \frac{\tilde{C}_{P}}{T_{0}}\left(T_{\mathrm{f}}-T_{\mathrm{i}}\right)-\frac{P_{0}}{T_{0}} \alpha n \tilde{V}\left(T_{\mathrm{f}}-T_{\mathrm{i}}\right) \\
- & \alpha n \tilde{V}\left(P_{\mathrm{f}}-P_{\mathrm{i}}\right)+\frac{P_{0}}{T_{0}} \beta n \tilde{V}\left(P_{\mathrm{f}}-P_{\mathrm{i}}\right) \\
& -\frac{\beta n \tilde{V}}{2 T_{0}}\left(P_{\mathrm{f}}^{2}-P_{\mathrm{i}}^{2}\right) .
\end{aligned}
$$

To the above we adjoin the line integrals

$$
\theta_{b d}=\frac{\alpha n \tilde{V}}{T_{0}}\left[\int_{0}^{\tau} P(t) \frac{\mathrm{d} T}{\mathrm{~d} t} \mathrm{~d} t+\int_{0}^{\tau} T(t) \frac{\mathrm{d} P}{\mathrm{~d} t} \mathrm{~d} t\right] .
$$

As was the case earlier, we verified that these also do not depend on the chosen time dependence; we thus adopted Case 1 , above, to carry out the integrations to find $\theta_{P}$ and $\theta_{T}$, which are summed to yield

$$
\theta_{b l}=\frac{\alpha n \tilde{V}}{T_{0}}\left(P_{\mathrm{f}} T_{\mathrm{f}}-P_{\mathrm{i}} T_{\mathrm{i}}\right) .
$$

The final result in the present case involves the sum of Eqs. (26) and (28). The only terms not referring to the expansion properties of the condensed phase are the first two in (26), which depend solely on the temperatures of the system and of the surroundings. Normally, the remaining terms are small compared to those just mentioned.

\section{Cyclic processes}

Up to now we have assumed a monotonic variation of temperature and pressure with time. Also of interest is a cyclic process in which the intensive variables of the system are changed in the sequence $T_{\mathrm{i}} \rightarrow T_{\mathrm{f}} \rightarrow T_{\mathrm{i}}$ and $P_{\mathrm{i}} \rightarrow P_{\mathrm{f}} \rightarrow P_{\mathrm{i}}$. For this purpose we attach to the system a reservoir at temperature $T_{1}<T_{\mathrm{i}}$ and pressure $P_{1}<P_{\mathrm{i}}$, while retaining the original reservoir at temperature $T_{0}>$ $T_{\mathrm{f}}$ and pressure $P_{0}>P_{\mathrm{f}}$. While the system interacts with reservoir 0 , reservoir 1 remains sealed off, and vice versa.

(a) In considering the case of the ideal gas the interaction with reservoir for the return process is handled by reversing the indices $i$ and $f$ and replacing subscript 0 by subscript 1 in (19) and (22). When these newly generated expressions are added to (19) and (22) one obtains for the cyclic process

$$
\begin{aligned}
\theta_{\mathrm{c}} & =\frac{3}{2} n R\left(\frac{1}{T_{1}}-\frac{1}{T_{0}}\right)\left(T_{\mathrm{f}}-T_{\mathrm{i}}\right)+n R\left(\frac{T_{\mathrm{i}}}{P_{\mathrm{i}}}-\frac{T_{\mathrm{f}}}{P_{\mathrm{f}}}\right) \\
& \times\left(\frac{P_{0}}{T_{0}}-\frac{P_{1}}{T_{1}}\right)=\frac{3}{2} n R\left(\frac{1}{T_{1}}-\frac{1}{T_{0}}\right)\left(T_{\mathrm{f}}-T_{\mathrm{i}}\right) \\
& +n R\left(\frac{1}{c_{i}}-\frac{1}{c_{\mathrm{f}}}\right)\left(c_{0}-c_{1}\right),
\end{aligned}
$$

where $c$ is the concentration of the gas phase. Thus, the second term drops out if the gas concentrations in the two reservoirs are rendered identical. In any case, the principal contribution is shown by the first term in (29), which agrees with the result cited in another derivation [7] where temperature and volume were taken as 
the independent variables. If the process had been carried out reversibly there would have been no change in entropy in the system.

(b) For the condensed phase a similar operation involving (26) and (28) leads to the result

$$
\begin{aligned}
\theta_{\mathrm{C}} & =-\left[n \tilde{\mathrm{c}}_{P}\left(\frac{1}{T_{0}}-\frac{1}{T_{1}}\right)+\alpha n \tilde{V}\left(\frac{P_{0}}{T_{0}}-\frac{P_{1}}{T_{1}}\right)\right] \\
& \times\left(T_{\mathrm{f}}-T_{\mathrm{i}}\right)+\alpha n \tilde{V}\left(\frac{1}{T_{0}}-\frac{1}{T_{1}}\right)\left(P_{\mathrm{f}} T_{\mathrm{f}}-P_{\mathrm{i}} T_{\mathrm{i}}\right) \\
& +\beta n \tilde{V}\left(\frac{P_{0}}{T_{0}}-\frac{P_{1}}{T_{1}}\right)\left(P_{\mathrm{f}}-P_{\mathrm{i}}\right)-\frac{\beta}{2} n \tilde{V}\left(\frac{1}{T_{0}}-\frac{1}{T_{1}}\right) \\
& \times\left(P_{\mathrm{f}}^{2}-P_{\mathrm{i}}^{2}\right) .
\end{aligned}
$$

Under ordinary laboratory conditions the quantities that involve $\beta \tilde{V}$ and $\alpha \tilde{V}$ will be small relative to $\tilde{C}_{P}$ and, in zero order approximation, may be ignored. Then the principal contribution to the entropy change of the condensed phase undergoing a cyclic process is given by

$$
\theta_{\mathrm{c}}=n \tilde{C}_{P}\left(T_{\mathrm{f}}-T_{\mathrm{i}}\right)\left(\frac{1}{T_{1}}-\frac{1}{T_{0}}\right) .
$$

\section{Discussion}

By eliminating the commonly used inequalities associated with the second law of thermodynamics we were able to relate differential elements of heat and work transfer carried out irreversibly to those executed reversibly. This permitted us to determine the differential form of the entropy associated with the irreversible transfer of heat and work across a thin boundary separating a reservoir from a system. The control variables governing the dependent variable were temperature, pressure, and composition. To apply these quantities to irreversible processes it was assumed that the latter occurred sufficiently slowly that in the system $T, P$, and $n_{i}$ could be altered uniformly over most of the physical extension of the system, but no limits were set between the difference of these quantities and the corresponding fixed ones of the reservoir.

As examples we considered ideal gases as well as condensed phases. When integrating we distinguished between integrals that involve either one or two variables. In the latter set we examined two cases with different time rates of change of temperature and pressure at constant compositions. As expected, the corresponding in- tegrals depend on the chosen path, but their sum is independent of the selection for the time variations of the independent variables. We also examined cyclic processes to note the net entropy increase in the universe upon irreversibly cycling the ideal gas or condensed phase.

These derivations should serve as a prototype for an analysis of irreversible phenomena. The present approach complements the standard theory of irreversible processes, in which emphasis is placed on the specification of fluxes, such as transport of entropy and matter, in response to external forces either within a system or across boundaries.

\section{Acknowledgments}

This research was supported through Award SI-06-021 by the Camille and Henry Dreyfus Foundation.

\section{References}

[1] Dor Ben-Amotz, J.M. Honig, J. Chem. Phys. 118 , 5932 (2003).

[2] J.M. Honig, Dor Ben Amotz, J. Chem. Ed. 83, 132 (2005).

[3] Dor Ben Amotz, J.M. Honig, Phys. Rev. Lett. 96, 020602 (2006).

[4] Dor Ben Amotz, J.M. Honig, J. Phys. Chem. B 110, 10066 (2006).

[5] J.M. Honig, Dor Ben Amotz, Chem. Educator 13, 220 (2008).

[6] J.M. Honig, Thermodynamics, Principles Characterizing Chemical and Physical Processes, 3rd ed., Academic Press, Amsterdam 2008.

[7] J.M. Honig, R. Hoehn, Open J. Chem. Thermo. 2011, in press.

[8] S.R. de Groot, P. Mazur, Non-Equilibrium Thermodynamics, North-Holland, Amsterdam 1962.

[9] I. Prigogine, Introduction to Thermodynamics of Irreversible Processes, Wiley, New York 1967.

[10] R. Haase, Thermodynamics of Irreversible Processes, Dover, New York 1990.

[11] R.C. Tolman, P.C. Fine, Rev. Mod. Phys. 20, 51 (1948).

[12] J. Kestin, A Course in Thermodynamics, Blaisdell, Waltham MA 1966, Ch. 13.

[13] A. Bejan, Advanced Engineering Thermodynamics, 2nd ed., Wiley, New York 1997, p. 135. 